\title{
Eficácia da mobilização articular em pacientes idosos com dor cervical crônica: uma
} revisão sistemática

\author{
Effectiveness of joint mobilization in elderly patients with chronic cervical pain: a systematic \\ review \\ Efectividad de la movilización articular en pacientes ancianos con dolor cervical crónico: una \\ revisión sistemática
}

Recebido: 09/11/2021 | Revisado: 15/11/2021 | Aceito: 16/11/2021 | Publicado: 26/11/2021

\author{
Antônia Neusa Lima Cardoso \\ ORCID: https://orcid.org/0000-0002-0199-2027 \\ Centro Universitário Santo Agostinho, Brasil \\ E-mail: neusa.lima.cardoso@gmail.com \\ Maria da Cruz Rodrigues da Silva Sousa \\ ORCID: https://orcid.org/0000-0003-4354-8896 \\ Centro Universitário Santo Agostinho, Brasil \\ E-mail: cruzsousa0209@gmail.com \\ Patrícia Lima Ventura \\ ORCID: https://orcid.org/0000-0002-8920-2877 \\ Centro Universitário Santo Agostinho, Brasil \\ E-mail: vlpatricia@hotmail.com
}

\begin{abstract}
Resumo
Objetivos: Identificar a eficácia da mobilização articular em pacientes idosos com dor cervical crônica. Métodos: O estudo foi baseado em uma revisão sistemática baseada no Preferred Reporting Items for Systematic Reviews and Meta-Analyses (PRISMA), foi conduzida nas seguintes bases de dados eletrônicas: PEDro (Physiotherapy Evidence Database), PubMed (National Library of Medicine, EUA) e a Central (a base de dados de ECAs da colaboração de Cochrane Libary). A pesquisa foi realizada utilizando os descritores: "Terapia manual". "Dor cervical", "Dor crônica", "Idoso" entre as palavras chaves com o propósito de otimizar os resultados e seus descritores em inglês: "Manual therapy", "Neck pain", "Chronic pain", "Aged" e o cruzamento das palavras através dos operadores booleanos " $A N D$ ” e “OR". Resultados: Os resultados demonstraram que a técnica de mobilização articular foram eficazes na melhora da dor cervical crônica. Conclusão: A técnica da mobilização articular em paciente idoso com dor cervical, comprova a diminuição da dor e aumento da capacidade funcional, proporcionando uma melhor qualidade de vida e apresentando eficácia à curto e longo prazo pela melhora do quadro clinico da dor cervical.
\end{abstract}

Palavras-chave: Terapia manual; Dor cervical; Dor crônica; Idoso.

\begin{abstract}
Objectives: To identify the effectiveness of joint mobilization in elderly patients with chronic neck pain. Methods: The study was based on a systematic review based on Preferred Reporting Items for Systematic Reviews and MetaAnalyses (PRISMA), conducted in the following electronic databases: PEDro (Physiotherapy Evidence Database), PubMed (National Library of Medicine, USA, USA) and Central (the Cochrane Libary Collaboration ECA Database). The research was carried out using the descriptors: "Manual therapy". "Cervical pain", "Chronic pain", "Elderly" among the keywords with the purpose of optimizing the results and their descriptors in English: "Manual therapy", "Neck pain", "Chronic pain", "Aged" and the crossing of the words through the Boolean operators "AND" and "OR". Results: The results showed that the joint mobilization technique was effective in improving chronic neck pain. Conclusion: The technique of joint mobilization in elderly patients with cervical pain, proves the decrease in pain and increase in functional capacity, providing a better quality of life and showing effectiveness in the short and long term by improving the clinical picture of cervical pain.
\end{abstract}

Keywords: Manual therapy; Neck pain; Chronic pain; Aged.

\section{Resumen}

Objetivos: Identificar la efectividad de la movilización articular en pacientes ancianos con dolor de cuello crónico. Métodos: El estudio se basó en una revisión sistemática basada en Preferred Reporting Items for Systematic Reviews and Meta-Analysis (PRISMA), realizado en las siguientes bases de datos electrónicas: PEDro (Physiotherapy Evidence Database), PubMed (National Library of Medicine, EE. UU., EE. UU. ) y Central (la base de datos ECA de la Colaboración Cochrane de Bibliotecas). La investigación se realizó utilizando los descriptores: "Terapia manual”. 
"Dolor cervical", "Dolor crónico", "Anciano" entre las palabras clave con el propósito de optimizar los resultados y sus descriptores en inglés: "Terapia manual", "Dolor de cuello", "Dolor crónico", "Anciano" y el cruce de las palabras a través de los operadores booleanos "Y" y "O". Resultados: Los resultados mostraron que la técnica de movilización articular fue eficaz para mejorar el dolor de cuello crónico. Conclusión: La técnica de movilización articular en pacientes ancianos con dolor cervical, demuestra la disminución del dolor y el aumento de la capacidad funcional, brindando una mejor calidad de vida y mostrando efectividad a corto y largo plazo al mejorar el cuadro clínico del dolor cervical.

Palabras clave: Terapia manual; Dolor de cuello; Dolor crónico; Anciano.

\section{Introdução}

O processo de envelhecimento é algo discutido historicamente; tendo em vista que a população nos tempos de cristo vivia por longos anos. Após isso a expectativa de vida fora diminuindo, porém, atualmente observa-se que a longevidade está voltando a fazer parte da vida das pessoas; ou seja, as pessoas idosas estão vivendo mais tempo; nota-se que a população está denotando uma diminuição nas taxas de fecundidade e elevando a expectativa de vida (Mari, Alves, Aerts, \& Camara, 2016).

É sabido que muitas vezes a velhice vem sendo concebida como um momento de constantes doenças como a dor cervical, porém, o envelhecimento é algo natural vivenciado por seres humanos, que, por ventura, pode ser uma etapa onde as pessoas tornam-se mais suscetíveis às doenças (Santos, Tonhom, \& Komatsu, 2016). Tendo em vista que o ser humano tende a considerar quaisquer alterações que possam surgir em sua saúde como intercorrências da idade (Biasus, 2016; Calaça, 2017).

Essas alterações causam consequências físicas como a redução da força muscular, da acuidade visual, da massa óssea e acarretam comprometimento neuromuscular. Como resultado desses fatores, os idosos estão mais suscetíveis a dor cervical, que é oriunda de disfunções musculoesqueléticas em região dorsal do pescoço e superior das escápulas, com ou sem irradiação para cabeça, tronco e membros superiores (Romero, Santana, Marques, Castanheira, Rodrigues, \& Sabbadini, 2018). O diagnóstico é feito através da identificação de sintomas como cefaleias, diminuição da amplitude de movimento da coluna cervical e torácica e dor irradiada para membros superiores (Silva, Costa, Soutinho, \& Pedrosa, 2017; Sprung, Lopes, 2016).

No que diz respeito à duração da dor cervical, pode ser classificada em aguda, quando há presença de dor súbita existente há menos de três meses; ou crônica, quando torna-se persistente. A etiologia da dor cervical pode ser bastante variável, desde causas menos comuns como doenças reumáticas, infecciosas e endócrinas, até as causas mais frequentes como: tensão, distúrbios posturais, espondilose cervical e causas traumáticas. Silva et al. (2017). Pourahmadi, Monhsenifar, Dariush, Aftabi, \& Amiri., (2018).

A dor crônica apresente de forma lenta, má localizada e difusa e de difícil tolerância, dura um longo período de tempo e pode variar de leve a severa, o que pode gerar uma condição altamente predominante e incapacitante que é frequentemente tratada com mobilizações passivaas (Park Hwang, 2021). Estima-se que o predomínio de dor cervical varia de $6 \%$ a $22 \%$ atingindo aproximadamente de 38\% da população idosa, com incidência maior no sexo feminino (Soares \& Campanholi, 2019). Dentre as inúmeras opções de terapêuticas conservadoras, a Fisioterapia tem comprovada efetividade no tratamento de dor cervical crônica, já que abrange uma vasta eficácia para prevenção e tratamento, e vem utilizando cada vez mais a terapia manual para as intervenções das afecções lombares, com destaque para as técnicas de mobilização articular e manipulação articular (Rauschkolb \& Gomes, 2016; Uthaikhup, Assapun, Watcharasaksilp, \& Jull., 2017).

Diante da patologia, entre as técnicas da fisioterapia manipulativa existe a técnica de mobilização articular Maitland (Tavares et al., 2017). Esse tipo de mobilização refere a movimentos passivos lentos, em grande amplitude, que visam recuperação das disfunções de movimentos artrocinemáticos, ou seja, melhora a congruência articular, diminui o atrito mecânico na articulação, diminui a dor, edema, e por consequência sua função biomecânica. Podem ser tanto movimentos acessórios como fisiológicos, ou combinados. A mobilização articular passiva tem sido uma técnica bastante utilizada nos tratamentos da dor cervical crônica, uma vez que grande parte das pessoas que tem sintomas agudos apresentam redução da mobilidade (KISNER, 2016). 
Uma vez que a técnica de mobilização articular tem sido uma técnica bastante utilizada nos tratamentos da dor cervical crônica em idosos, devido grande parte dessa população apresentar sintomas agudos e consequentemente redução da mobilidade (Oliveira, Peres, Bellini, \& Campos., 2018).

Dessa forma, o presente estudo objetiva esclarecer e reunir referências acerca da eficácia da mobilização articular em pacientes idosos com dor cervical crônica visando responder a pergunta norteadora: "A mobilização articular é efetiva na dor cervical crônica em idosos?”. Para responder tal pergunta, parte da estratégia PICO (population/população; intervention/intervenção; comparison/comparação e outcome/desfecho).

"P" Idoso com dor cervical;

"I" Mobilização articular;

"C" Nenhuma intervenção;

"O" Efetividade da mobilização articular na dor cervical crônica em idosos.

Com isso o presente estudo tem importância para que o conhecimento seja implementado cada vez mais no tratamento dos idosos com dor cervical crônica e enriqueça a literatura sobre a mobilização articular como técnica da Fisioterapia no tratamento da patologia.

\section{Metodologia}

Uma busca sistemática da literatura foi redigida, baseada noPreferred Reporting Items for Systematic Reviews and Meta-Analyses (PRISMA), que foi conduzida nas seguintes bases de da dos eletrônicas: PEDro (Physiotherapy Evidence Database), PubMed (National Library of Medicine, EUA) e a Central (a base de dados de ECAs da colaboração de Cochrane Libary). A pesquisa foi realizada utilizando os descritores: "Terapia manual". "Dor cervical", "Dor crônica", "Idoso" entre as palavras chaves com o propósito de otimizar os resultados e seus descritores em inglês: "Manual therapy", "Neck pain", "Chronic pain", “Aged" e o cruzamento das palavras através dos operadores booleanos " $A N D$ " e "OR". Dois investigadores foram responsáveis por essa etapa de pesquisa, que foi realizada no período de agosto a outubro de 2021.

Segundo Sampaio e Mancini (2017), além de, sistematizar, reúnir os dados de estudos, e produzir considerações sobre o que a literatura informa sobre a alguma intervenção específica, mediante a uma sistematizada de busca, de uma aplicação de uma metodologia explícita, uma síntese de informação selecionada e apreciação crítica. Sendo que permite a síntese de vários estudos publicados e permite conclusões a respeito de um estudo específico (Beck e Lipincott, 2006).

Os artigos científicos necessitam satisfazer a alguns critérios de inclusão e foram coordenados por meio da estratégia PICO, tendo como questão norteadora: “A mobilização articular é efetiva na dor cervical crônica em idosos?” Nesta pergunta, o primeiro elemento da estratégia $(\mathrm{P})$ refere-se ao Idoso com dor cervical crônica; "I" à mobilização articular; "C" à nenhuma intervenção; "O” refere-se a efetividade da mobilização articular na dor cervical crônica em idosos.

Foram selecionados estudos com os seguintes critérios de inclusão: participantes idosos, idosos com queixa de dor cervical crônica, a mobilização articular como tratamento da dor cervical crônica dos pacientes idosos. Foram excluídos os artigos que não envolviam a população idosa que não tinha o diagnóstico de dor cervical crônica, estudos que não utilizava a mobilização articular como tratamento da dor cervical crônica. Referências de artigos pertinentes também foram pesquisados sem nenhuma restrição de tempo e de idioma. Toda organização e distribuição do número de registros encontrados, selecionados e excluídos, foram dispostos no fluxograma PRISMA, Figura 1.

Com intuito de aumentar a confiabilidade na seleção dos artigos, os estudos foram potencialmente revisados por dois pesquisadores que entraram em comum acordo para estabelecer quais artigos preenchiam os critérios de inclusão. Estudos cujos resumos, não continham informações precisas que permitisse a inclusão não foram selecionados. Seguindo os mesmos critérios 
de elegibilidade previamente definidos após leitura dos artigos na íntegra foi determinado a inclusão dos artigos para os resultados, o qual, foi realizada a síntese das evidências, que compreende a extração dos elementos a seguir: título do artigo, autor/ano, objetivos, delineamento do estudo, intervenção, resultados significativos.

Todos os dados extraídos estão dispostos na Tabela 2 a fim de facilitar a compreensão do leitor. A informação extraída incluiu a avaliação do risco de viés através da Escala PEDro que foi utilizada para avaliar a qualidade dos estudos conforme Tabela 1. Essa escala possui onze itens, cada um deles contribui com 1 ponto, com exceção do primeiro, assim a pontuação pode variar de 0 a 10 na qual sua contagem é determinada pela soma dos itens que tenham sido compridos no ensaio clínico.

\section{Resultados}

A busca nos bancos de dados resultou em 461 estudos. Após a exclusão de 61 artigos em duplicidade, 400 artigos foram realizados a leitura dos títulos e resumos e 380 destes artigos forma excluídos por critérios de elegibilidade por estarem incompletos (68), não apresentaram participantes idosos (139), não adotaram a mobilização articular como tratamento da dor cervical crônica (107) e não avaliaram os desfechos desejados (66).

Sendo assim, foram selecionados 20 artigos para leitura do texto na íntegra, no final 14 foram excluídos por não está dentro dos critérios de elegibilidade dessa revisão. Na Figura 1 apresenta as etapas de elegibilidades dos artigos, onde foram incluídos um total de 06 estudos para composição da pesquisa (Figura 1).

Figura 1 - Fluxograma das etapas de elegibilidades dos artigos pesquisados e utilizados para os resultados.

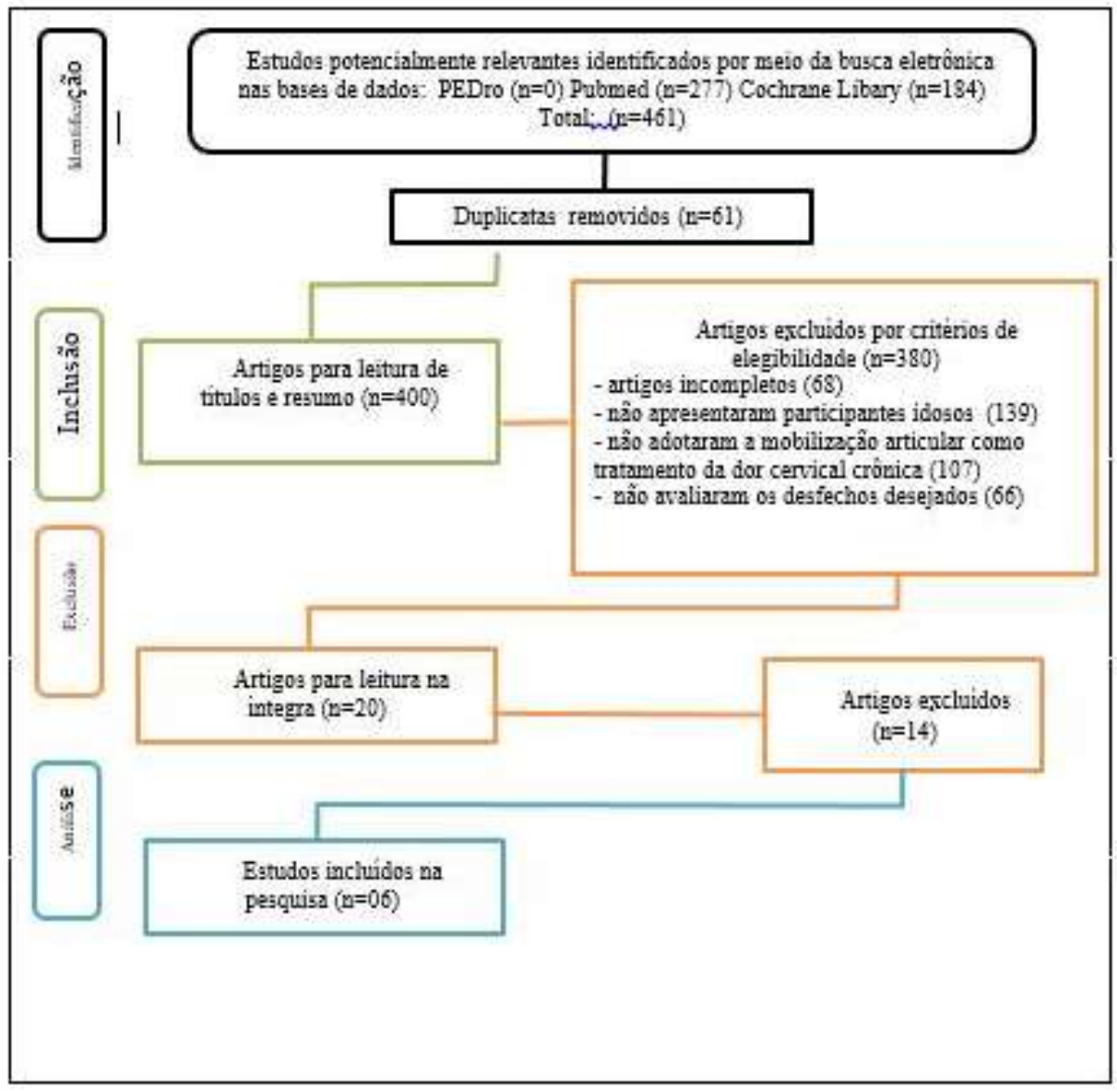

Fonte: PRISMA (2009). 
Na Figura 1 apresenta os critérios de elegibilidade dos artigos de inclusão e exclusão do presente estudo.

A seguir apresenta a Tabela 1 onde contém a análise da qualidade metodológica dos estudos por meio da escala PEDRo, contendo os critérios de elegibilidade, distribuição aleatória, alocação secreta dos sujeitos, semelhança inicial entre os grupos, cegamentos dos sujeitos, cegamentos dos avaliadores, acompanhamento adequado, análise da intenção de tratamento, comparação inter-grupos, medidas de precisão e variabilidade, com pontuações de 1 para itens contemplados e 0 para itens não contemplados.

Tabela 1: Análise da qualidade metodológica dos estudos por meio da escala PEDRo.

\begin{tabular}{|c|c|c|c|c|c|c|}
\hline Escala PEDRo & $\begin{array}{l}\text { Buyukturan, et } \\
\text { al... (2018) }\end{array}$ & $\begin{array}{l}\text { Maiers, et } \\
\text { al.,. (2019) }\end{array}$ & $\begin{array}{l}\text { Gautam; } \\
\text { Dhamija; } \\
\text { Puri (2014) }\end{array}$ & $\begin{array}{l}\text { Kim et al., } \\
\text { (2015) }\end{array}$ & $\begin{array}{l}\text { Shin; } \\
\text { Choi } \\
(2010)\end{array}$ & $\begin{array}{l}\text { Aquino et } \\
\text { al., (2009) }\end{array}$ \\
\hline Critérios de Elegibilidade & Sim & Sim & Sim & Sim & Sim & Sim \\
\hline $\begin{array}{c}\text { Distribuição } \\
\text { Aleatória }\end{array}$ & 1 & 1 & 1 & 1 & 1 & 1 \\
\hline Alocação secreta dos sujeitos & $\mathbf{0}$ & $\mathbf{0}$ & $\mathbf{0}$ & $\mathbf{0}$ & $\mathbf{0}$ & $\mathbf{0}$ \\
\hline $\begin{array}{c}\text { Semelhança Inicial entre os } \\
\text { grupos }\end{array}$ & 1 & 1 & 1 & $\mathbf{0}$ & $\mathbf{0}$ & 1 \\
\hline Cegamentos dos sujeitos & 1 & 1 & 1 & 1 & 1 & 1 \\
\hline Cegamentos dos terapeutas & $\mathbf{0}$ & $\mathbf{0}$ & 1 & $\mathbf{0}$ & $\mathbf{0}$ & $\mathbf{0}$ \\
\hline Cegamentos dos avaliadores & $\mathbf{0}$ & 1 & 1 & 1 & 1 & 1 \\
\hline Acompanhamento adequado & 1 & $\mathbf{0}$ & $\mathbf{0}$ & 1 & 1 & 1 \\
\hline $\begin{array}{l}\text { Análise da intenção de trata- } \\
\text { mento }\end{array}$ & 1 & 1 & 1 & 1 & 1 & 1 \\
\hline Comparação intergrupos & 1 & 1 & 1 & $\mathbf{0}$ & 1 & 1 \\
\hline $\begin{array}{l}\text { Medidas de precisão e variabili- } \\
\text { dade }\end{array}$ & 1 & 1 & 1 & 1 & 1 & 1 \\
\hline Escore Total & $7 / 10$ & $6 / 10$ & 8/10 & 6/10 & $7 / 10$ & $8 / 10$ \\
\hline
\end{tabular}

* Segundo a escala PEDRo, sendo 1 para itens contemplados e 0 para itens não contemplados. Fonte: Autores.

Na Tabela 1 apresenta a pontuação dos artigos e análise metodológica de acordo com a escala PEDro, sendo 1 para itens contemplados e 0 para itens não contemplados.

Na Tabela 2 apresenta os artigos contendo a produção científica e a relação quanto ao título do artigo, autor/ano, objetivos, delineamento do estudo, intervenção e resultados significativos. 


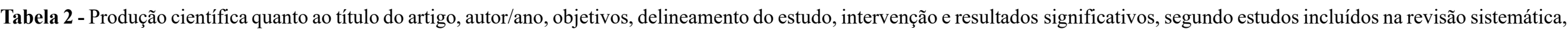
Teresina - PI, Brasil, 2021.

\begin{tabular}{|c|c|c|c|c|c|}
\hline $\begin{array}{l}\text { Título do ar- } \\
\text { tigo }\end{array}$ & Autor/Ano & Objetivos & $\begin{array}{l}\text { Delinea- } \\
\text { mento do estudo }\end{array}$ & Intervenção & Resultados Significativos \\
\hline $\begin{array}{l}\text { O efeito da técnica } \\
\text { de mobilização de } \\
\text { Mulligan em adul- } \\
\text { tos mais velhos } \\
\text { com dor no pes- } \\
\text { coço: um estudo } \\
\text { duplo-cego contro- } \\
\text { lado randomizado }\end{array}$ & $\begin{array}{l}\text { Buyukturan, } \\
\text { et al.,. (2018) }\end{array}$ & $\begin{array}{l}\text { examinar o efeito da téc- } \\
\text { nica de mobilização de Mulli- } \\
\text { gan (MMT) na dor, amplitude } \\
\text { de movimento (ROM), nível } \\
\text { funcional, cinesiofobia, depres- } \\
\text { são e qualidade de vida (QV) } \\
\text { em idosos com dor no pescoço } \\
\text { ( NP). }\end{array}$ & $\begin{array}{l}\text { Estudo Randomi- } \\
\text { zado }\end{array}$ & $\begin{array}{l}\text { Intervenção: Quarenta e dois idosos que concordaram foram dividi- } \\
\text { dos em dois grupos usando um método de randomização pareada. To- } \\
\text { dos os participantes do grupo de fisioterapia tradicional (TP) e grupo } \\
\text { fisioterapia tradicional-mobilização Mulligan (TPMM). O programa } \\
\text { de tratamento foi agendado para } 10 \text { sessões. Os participantes foram } \\
\text { avaliados em termos de dor, Amplitude do Movimento (ADM), nível } \\
\text { funcional, cinesiofobia, depressão e QV tanto pré quanto pós-trata- } \\
\text { mento. } \\
\text { Controle: Os participantes foram informados e educados sobre for- } \\
\text { mas eficazes de realizar suas atividades de vida diária. }\end{array}$ & $\begin{array}{l}\text { Dor, ADM, nível funcional, cinesiofobia, depres- } \\
\text { são e QV melhoraram em ambos os grupos após o tra- } \\
\text { tamento . Ao comparar os efeitos desses dois progra- } \\
\text { mas de tratamento, observou-se que o grupo TPMM } \\
\text { teve um melhor resultado em termos de ADM, cinesio- } \\
\text { fobia, depressão e QV }\end{array}$ \\
\hline $\begin{array}{l}\text { Tratamento de } \\
\text { curto ou longo } \\
\text { prazo para defici- } \\
\text { ência da coluna } \\
\text { vertebral em adul- } \\
\text { tos mais velhos } \\
\text { com manipulação } \\
\quad \text { e exercício }\end{array}$ & $\begin{array}{l}\text { Maiers, et } \\
\text { al.,. (2019) }\end{array}$ & $\begin{array}{l}\text { Comparar a eficácia da te- } \\
\text { rapia manipulativa espinhal } \\
\text { (SMT) combinada com exercí- } \\
\text { cios de reabilitação supervisio- } \\
\text { nados (SRE). }\end{array}$ & $\begin{array}{l}\text { Ensaio Clínico } \\
\text { Randomizado }\end{array}$ & $\begin{array}{l}\text { Intervenção: comparou o tratamento de curto prazo (12 semanas) } \\
\text { versus gerenciamento de longo prazo ( } 36 \text { semanas) de deficiência re- } \\
\text { lacionada às costas e pescoço em idosos adultos usando terapia mani- } \\
\text { pulativa espinhal (SMT) combinada com exercícios de reabilitação } \\
\text { supervisionados. } \\
\text { Controle: Não recebeu nenhum tipo de intervenção }\end{array}$ & $\begin{array}{l}182 \text { participantes foram randomizados. Os grupos } \\
\text { de curto e longo prazo demonstraram melhorias signi- } \\
\text { ficativas nas costas }(-3,9 \text {, intervalo de confiança de } \\
95 \% \text { (IC) }-5,8 \text { a }-2,0 \text { contra }-6,3 \text {, IC } 95 \%-8,2 \text { a } \\
-4,4) \text { e deficiência cervical }(-7,3 \text {, IC de } 95 \%-9,1 \text { a } \\
-5,5 \text { versus }-9,0 \text {, IC de } 95 \%=-10,8 \text { a }-7,2) \text { após } 36 \\
\text { semanas, sem diferença entre os grupos (costas } 2,4, \text { IC } \\
\text { de } 95 \% \text { - } 0,3 \text { a } 5,1 \text { pescoço } 1,7 \text {, IC de } 95 \% \text { - } 0,8 \text { a } \\
4,2 \text { ). O grupo de tratamento de longo prazo experi- } \\
\text { mentou maior melhora na dor no pescoço na semana } \\
\text { 36, autoeficácia nas semanas } 36 \text { e } 52 \text {, capacidade fun- } \\
\text { cional e equilíbrio. }\end{array}$ \\
\hline $\begin{array}{l}\text { Comparação } \\
\text { da mobilização da } \\
\text { Maitland e Mulli- } \\
\text { gan na melhoria da } \\
\text { dor pescoço, rom e } \\
\quad \text { deficiência }\end{array}$ & $\begin{array}{l}\text { Gautam; Dha- } \\
\text { mija; Puri (2014) }\end{array}$ & $\begin{array}{l}\text { Comparar a eficácia de dois } \\
\text { diferentes técnicas de } \\
\text { mobilização para melhorar a } \\
\text { dor, amplitude de movimento - } \\
\text { ROM e deficiência }\end{array}$ & $\begin{array}{l}\text { Estudo Randomi- } \\
\text { zado }\end{array}$ & $\begin{array}{l}\text { Intervenção: Um total de } 30 \text { indivíduos foram tomados e divididos } \\
\text { aleatoriamente em três grupos: Grupo A, grupo B, grupo C (cada } \\
\text { grupo com } 10 \text { sujeitos). O grupo A estava sob terapia convencional. } \\
\text { Grupo B com as técnicas de mobilização de Maitland e o grupo C } \\
\text { com a técnica de mobilização de Mulligan. } \\
\text { Controle: Não recebeu nenhum tipo de intervenção }\end{array}$ & $\begin{array}{l}\text { Este estudo mostrou que a mobilização de mulligan é } \\
\text { mais eficaz na melhora da dor, amplitude do movi- } \\
\text { mento - ADM e incapacidade. Embora ambos os gru- } \\
\text { pos experimentais tenham mostrado diminuição na } \\
\text { dor, deficiência e melhora da ROM, mas a mobilização } \\
\text { de Mulligan foi considerada mais eficaz na melhora } \\
\text { dor, ADM e deficiência. }\end{array}$ \\
\hline $\begin{array}{l}\text { Efeitos da técnica } \\
\text { de liberação ativa } \\
\text { na dor e amplitude } \\
\text { de movimento de } \\
\text { pacientes com dor } \\
\text { cervical crônica }\end{array}$ & Kim et al., (2015) & $\begin{array}{l}\text { Comparar as influências da téc- } \\
\text { nica de liberação ativa (ART) e } \\
\text { mobilização articular (JM) na } \\
\text { escala visual analógica (VAS), } \\
\text { pontuação de dor, limiar de dor } \\
\text { à pressão (PPT) e amplitude de } \\
\text { movimento cervical (ADM) de }\end{array}$ & $\begin{array}{l}\text { Estudo Randomi- } \\
\text { zado }\end{array}$ & $\begin{array}{l}\text { Intervenção: Os } 24 \text { sujeitos com dor cervical crônica incluídos no } \\
\text { estudo foram aleatoriamente designados a um de três grupos se- } \\
\text { guindo um projeto de pré-teste/pós-teste de grupo de controle equiva- } \\
\text { lente. Para } 3 \text { semanas, os grupos ART e JM receberam tratamento } \\
\text { duas vezes por semana durante } 20 \text { minutos. }\end{array}$ & $\begin{array}{l}\text { A ART foi a melhor opção para o tratamento de paci- } \\
\text { entes com dor cervical crônica no ambiente clínico. }\end{array}$ \\
\hline
\end{tabular}




\begin{tabular}{|c|c|c|c|c|c|}
\hline & & $\begin{array}{l}\text { pacientes com dor cervical crô- } \\
\text { nica. }\end{array}$ & & $\begin{array}{l}\text { Controle: Depois que todas as intervenções foram concluída, a pon- } \\
\text { tuação VAS, PPT e ROM foram medidos novamente. }\end{array}$ & \\
\hline $\begin{array}{l}\text { Efeitos de } \\
\text { exercícios especí- } \\
\text { ficos na dor cervi- } \\
\text { cal crônica em ido- } \\
\text { sos }\end{array}$ & Shin; Choi (2010) & $\begin{array}{l}\text { Identificar os efeitos de exercí- } \\
\text { cios específicos em mulheres } \\
\text { idosas com dor cervical crônica } \\
\text { (CNP) }\end{array}$ & $\begin{array}{l}\text { Estudo Randomi- } \\
\text { zado }\end{array}$ & $\begin{array}{l}\text { Intervenção: } 29 \text { idosas com queixa de CNP foram classificadas em } \\
\text { grupo experimental (14) e grupo controle (15). O grupo experimen- } \\
\text { tal foi tratado com exercícios específicos e terapia convencional mí- } \\
\text { nima, enquanto o grupo controle foi tratados apenas com terapia con- } \\
\text { vencional. Em seguida, os efeitos na escala visual analógica (VAS), } \\
\text { índice de deficiência do pescoço (NDI), escala de ansiedade de auto- } \\
\text { avaliação (SAS), forma coreana de escala de depressão geriátrica } \\
\text { (KGDS) e amplitude de movimento (ROM) foram comparados entre } \\
\text { os dois grupos. } \\
\text { Controle: O exercício específico para retreinar a função muscular da } \\
\text { coluna cervical é preconizado no tratamento da dor cervical. }\end{array}$ & $\begin{array}{l}\text { O grupo experimental mostrou resultados significati- } \\
\text { vamente melhorados em VAS, NDI, SAS, KGDS e } \\
\text { ROM após a intervenção }(p<0,05) \text {, assim como o } \\
\text { grupo controle }(\mathrm{p}<0,05) \text {. A comparação das mudanças } \\
\text { no grupo experimental antes e depois intervenção mos- } \\
\text { trou resultados superiores nos resultados SAS, NDI e } \\
\text { ROM (flexão, rotação, lat. flexão) quando comparados } \\
\text { com o grupo controle ( } \mathrm{p}<0,05) \text {. }\end{array}$ \\
\hline $\begin{array}{c}\text { A aplicação da } \\
\text { mobilização arti- } \\
\text { cular em diferentes } \\
\text { níveis vertebrais } \\
\text { cervicais não in- } \\
\text { fluencia a redução } \\
\text { imediata da dor em } \\
\text { pacientes com dor } \\
\text { cervical crônica: } \\
\text { um ensaio clínico } \\
\text { randomizado }\end{array}$ & $\begin{array}{l}\text { Aquino et al., } \\
\text { (2009) }\end{array}$ & $\begin{array}{l}\text { Comparar os efeitos da aplica- } \\
\text { ção da junta } \\
\text { mobilização em níveis cer- } \\
\text { vicais sintomáticos e assintomá- } \\
\text { ticos em pacientes com cervi- } \\
\text { calgia crônica inespecífica. }\end{array}$ & $\begin{array}{l}\text { Estudo Randomi- } \\
\text { zado }\end{array}$ & $\begin{array}{l}\text { Intervenção: } 48 \text { idosos com mais de } 65 \text { anos foram incluídos foram } \\
\text { randomizados para um de dois grupos de tratamento: (i) grupo con- } \\
\text { trole: o nível vertebral mais sintomático foi mobilizado; (ii) grupo } \\
\text { experimental: um nível vertebral selecionado aleatoriamente foi esco- } \\
\text { lhido e mobilizado. Todos os pacientes receberam uma sessão de tra- } \\
\text { tamento. A intensidade da dor na posição de repouso durante o movi- } \\
\text { mento cervical ativo mais doloroso, bem como durante a palpação } \\
\text { vertebral, foi quantificada por meio de uma escala de dor de } 11 \text { pon- } \\
\text { tos. } \\
\text { Controle: Medidas de acompanhamento foram tomadas imediata- } \\
\text { mente após a intervenção de um avaliador cego. }\end{array}$ & $\begin{array}{l}\text { Mudança significativa na intensidade da dor imediata } \\
\text { durante o movimento ativo doloroso e palpação verte- } \\
\text { bral foi alcançada após a mobilização vertebral. no en- } \\
\text { tanto, ambos os grupos apresentaram reduções de dor } \\
\text { semelhantes, sugerindo que a redução da dor devido à } \\
\text { mobilização articular não é específica ao nível verte- } \\
\text { bral a ser mobilizado. }\end{array}$ \\
\hline
\end{tabular}

Fonte: Autores (2021).

Na Tabela 2 apresenta a produção científica quanto ao título do artigo, autor/ano, objetivos, delineamento do estudo, intervenção e resultados significativos. 


\section{Discussão}

O Maiers et al., (2019) investigaram a eficácia relativa do tratamento de curto prazo (12 semanas) versus gerenciamento de longo prazo (36 semanas) de tratamentos não farmacológicos comumente recomendados, Terapia Manipulativa Espinhal (SMT) combinada com Exercícios de Reabilitação Supervisionados (SRE), para idosos que sofrem de dor lombar e cervical. Enquanto ambos os grupos experimentaram melhorias na deficiência desde o início até a semana 36, não houve diferenças estatisticamente significativas e pequeno tamanho do efeito entre os grupos (tamanho do efeito de Cohen de 0,22 para NDI e 0,25 para ODI). Ambos os grupos alcançaram a maior melhora média no momento da conclusão do tratamento e, geralmente, melhora sustentada em longo prazo.

As expectativas demonstraram ser preditivas de incapacidade persistente entre idosos recebendo tratamento para doenças musculoesqueléticas. Inicialmente, as expectativas de melhoria eram maiores entre os participantes randomizados para o grupo de manejo de longo prazo, em comparação com aqueles que receberam um curso mais curto de tratamento. Como resultado, essa diferença de linha de base foi incluída como uma covariável na análise. Ao longo do estudo de Maiers et al., (2019), ambos os grupos reduziram suas expectativas de melhora, ainda mais no grupo de tratamento de curto prazo. As razões para essa mudança nas expectativas e seu impacto na melhoria justificam uma exploração mais aprofundada.

Esse efeito foi ao contrário no estudo de Aquino et al., (2019) que apontaram que as mobilizações da articulação cervical produzem redução imediata da dor durante o movimento e palpação em pacientes com dor crônica no pescoço. Foi notado um alívio significativo da dor pós-tratamento durante o mais doloroso movimento ativo e palpação vertebral para ambos os grupos. Essas descobertas sugerem que os pacientes com dor crônica no pescoço pode sentir um alívio imediato da dor durante o movimento e palpação vertebral, independentemente de qual vértebra cervical nível está sendo mobilizado. Demonstrando que As mobilizações da articulação cervical produzem redução imediata da dor durante o movimento e palpação em pacientes com dor crônica no pescoço. No entanto, esses efeitos não são influenciados pelo quadro cervical segmento sendo mobilizado.

Em relação ao tempo de tratamento, Gautam, Dhamija, \& Puri (2014), enfatiza o que foi administrado 4 vezes por semana em um total de 30 dias. Dor, deficiência e ADM foram avaliados por irradiação numérica de dor escala, NDI e goniômetro universal. A avaliação foi feita aos 0, 15 e 30 dias de tratamento. A significância estatística foi estabelecida ao nível de 5\%. Este estudo mostrou que a mobilização de mulligan é mais eficaz na melhoria da dor, ROM e Deficiência embora ambos os grupos experimentais (grupo B e C) mostraram diminuição da dor e deficiência, mas o grupo C mostrou significante diminuição da dor e deficiência e melhora.

Buyukturan, Buyukuturan, Sas, Kararti, \& Ceylan (2018), identifica em termos de população de pacientes, que foi obtido maiores ganhos na QV nos participantes. O grupo fisioterapia tradicional-mobilização Mulligan (TPMM) que é uma técnica que se baseia na mobilização articular associada ao movimento que contribuiu para a maior amplitude do movimento $(\mathrm{ADM})$ e redução da dor, ambos os quais têm efeitos positivos na Qualidade de Vida (QV). Esses dois parâmetros são ganhos mais significativamente no grupo TPMM. Afirma-se também que, no caso de dor cervical crônica, a ADM da região cervical diminui, os movimentos são mais lentos que o normal e a propriocepção fica prejudicada. Acredita-se que qualquer aumento na ADM resulte em um aumento na sensação proprioceptiva na região do pescoço, o que pode resultar em redução da cinesofobia nos pacientes.

Da mesma forma, que Shin \& Choi (2010), evidenciaram que os pacientes realizaram exercícios de mobilização articular, juntamente com exercício de flexão crânio-cervical (CCFE) que trouxe sobre mudanças significativas no CROM ( $\mathrm{p}<0,05)$. O exercício específico para retreinar a função muscular da coluna cervical é preconizado no tratamento da dor cervical. Esta intervenção demonstrou que melhorou o controle temporal dos músculos flexores cervicais profundos e propriocepção do pescoço, além de levar a alterações na dor e deficiência em distúrbios cervicais dolorosos. 
Embora a maioria dos estudos evidenciam reduções da dor devido à mobilização articular. Kim, Lee, \& Park (2015) ao avaliar a eficácia do Mobilização Articular (JM) realizou um comparativo com a técnica de liberação ativa (ART) e a (JM), verificou-se que são ambos eficazes para o tratamento de pacientes com dor cervical crônica, mas no estudo em evidência apontou que a ART demonstraram uma tendência de maior eficácia para pacientes com dor cervical envolvendo lesão de tecidos moles. Este efeito maior pode estar relacionado à observação de que a lesão do tecido mole é a causa da dor em $87,5 \%$ dos casos de dor no pescoço, e ART é realizada diretamente no tecido mole lesado, enquanto JM trata a área limitada da junta. No entanto, a manipulação articular realizada na faixa final da amplitude do movimento do pescoço diretamente nas articulações das vértebras cervicais pode causar tensão nos músculos do pescoço do paciente, porque a região cervical vértebras são a parte mais sensível da coluna e esta a tensão protege os nervos e os vasos sanguíneos.

\section{Conclusão}

Conforme os resultados obtidos no presente estudo de revisão, podemos ratificar os benefícios da técnica da mobilização articular em pacientes idosos com dor cervical crônica, que comprovam a diminuição da dor e aumento da capacidade funcional, proporcionando assim uma melhor qualidade de vida aos indivíduos envolvidos, apresentando eficácia à curto e longo prazo sendo confirmada pela melhora do quadro clinico da dor cervical.

Apesar dos benefícios que a técnica da mobilização articular proporciona, ainda são necessários mais estudos devido à escassez em relação a aplicação da técnica, especificamente na dor cervical crônica em idoso, sendo assim, é notório a limitação do estudo diante da necessidade de mais ensaios clínicos com melhor descrição da técnica aplicada, para trazer mais evidências da eficácia, além de auxiliar profissionais que lidem com o público-alvo da pesquisa visando sempre a melhor conduta durante a problemática tratada.

\section{Referências}

Aquino, R. L., Caires, P. M., Furtado, F. C., Loureiro, A. V., Ferreira, P. H., \& Ferreira, M. L. (2009). Applying Joint Mobilization at Different Cervical Vertebral Levels does not Influence Immediate Pain Reduction in Patients with Chronic Neck Pain: A Randomized Clinical Trial. The Journal of manual \& manipulative therapy, 17 (2), 95-100. doi.org/10.1179/10669810979082468.

Beck, C. T., \& Lipincott. W. (2006). Fundamentos da pesquisa em enfermagem: métodos, avaliação e utilização. Nurse researcher, 13(4), 91-92. https://doi.org/10.7748/nr.13.4.91.s11

Biasus, F. (2016). Reflexões sobre o envelhecimento humano: aspectos psicológicos e relacionamento familiar. Perspectiva, Erechim. v. 40, n.152, p. 55-63.

Buyukturan, O., Buyukturan, B., Sas, S., Karartı, C., \& Ceylan, İ. (2018). The Effect of Mulligan Mobilization Technique in Older Adults with Neck Pain: A Randomized Controlled, Double-Blind Study. Pain research \& management, 2018, 2856375.

doi.org/10.1155/2018/2856375

Calaça, Frederico Igor Ribeiro. (2017). Influência da Técnica Mobilização com Movimento e Tape de Mulligan em indivíduos com instabilidade funcional de tornozelo: ensaio clínico randomizado e cego. Dissertação de mestrado em Ciências e Tecnologias em Saúde —Universidade de Brasília, Brasília, 35f.

Gautam, R., Dhamija, JK, \& Puri, A. (2014). COMPARAÇÃO DA MOBILIZAÇÃO DA MAITLAND E MULLIGAN NA MELHORIA DA DOR PESCOÇO, ROM E DEFICIÊNCIA. Jornal internacional de fisioterapia e pesquisa, 2 , 482-487.

Kim, JH, Lee, HS, \& Park, SW (2015). Efeitos da técnica de liberação ativa na dor e amplitude de movimento de pacientes com dor cervical crônica. Journal of Physical Therapy Science, 27 (8), 2461-2464.doi.org/10.1589/jpts.27.2461

Kisner, Carolyn (2016). Exercícios terapêuticos: fundamentos e técnicas. 6. Ed. Barueri-SP: Manole.

Maiers, M., Hartvigsen, J., Evans, R., Westrom, K., Wang, Q., Schulz, C., Leininger, B., \& Bronfort, G. (2019). Short- or Long-Term Treatment of Spinal Disability in Older Adults With Manipulation and Exercise. Arthritis care \& research, 71(11), 1516-1524.doi.org/10.1002/acr.23798

Mari, F, Alves, Gehysa, Aerts, Denise, Camara, Sheila. (2016). O processo de envelhecimento e a saúde: o que pensam as pessoas de meia-idade sobre o tema. Rev. Bras. Geriatr. Gerontol., Rio de Janeiro, v. 19, n. 1, p. 35-44. doi.org/10.1590/1809-9823.2016.14122

Oliveira, S.D.; Peres, C.M.; Bellini, P.E.; Campos, M.F. (2018). Tratamento da cervicalgia crônica com fisioterapia convencional - estudo de caso. Revista Cientifica do Centro Universitário de Itaipira, v. 03, n. 01, p. 140-152.

Park, D. J., Hwang, Y. I. (2021). Correlations between the Respiratory Function and Cervical and Lumbar Range of Motion in Elderly People.| J Korean Soc Phys Med.,v. 16, n.3. doi.org/10.13066/kspm.2021.16.3.29 
Research, Society and Development, v. 10, n. 15, e283101523037, 2021

(CC BY 4.0) | ISSN 2525-3409 | DOI: http://dx.doi.org/10.33448/rsd-v10i15.23037

Pourahmadi, M. R., Mohsenifar, H., Dariush, M., Aftabi, A., \& Amiri, A. (2018). Effectiveness of mobilization with movement (Mulligan concept techniques) on low back pain: a systematic review. Clinical rehabilitation, 32(10), 1289-1298. doi.org/10.1177/0269215518778321

Rauschkolb, P.; Gomes. T.N. (2016). Efeitos das técnicas manuais de mobilização e manipulação articulares da coluna vertebral. Revista Saúde Integrada, v. 9 , n. 17, p. 2-8. Retrieved from http://local.cnecsan.edu.br/revista/index.php/saude/index.

Romero, D. E., Santana, D., Marques, A., Castanheira, D., Rodrigues, J. M., \& Sabbadini, L., (2018). Prevalência, fatores associados e limitações relacionados ao problema crônico de coluna entre adultos e idosos no Brasil. Cad. Saúde Pública, v.34.n.2, p.12-81. doi.org/10.1590/0102-311X00012817

Sampaio, R. F., \& Mancini, M. C. (2007). Estudos de revisão sistemática: um guia para síntese criteriosa da evidência científica. Braz. J. Phys. Ther. 11 (1). doi.org/10.1590/S1413-35552007000100013

Santos, S. de C., Tonhom, S. F. da R., Tonhom, S. F. da R., Komatsu, R. S., \& Komatsu, R. S. (2017). Saúde do idoso: reflexões acerca da integralidade do cuidado. Revista Brasileira Em Promoção Da Saúde, 29, 118-127. doi.org/10.5020/18061230.2016.sup.p118

Shin, S. H., Choi, J. D. (2010). Effects of Specific Exercise on Chronic Neck Pain in Elderly Women. J Kor Soc Phys Ther 2010;22(3):1-8. Retrieved from https://scienceon.kisti.re.kr/srch/selectPORSrchArticle.do?cn=JAKO201031559286707

Silva AF, Costa MAL, Soutinho RSR, \& Pedrosa AS. (2017). Prevalência de cervicalgia em acadêmicos de odontologia de um Centro Universitário. Revist. Port.: Saúde e Sociedade, v.2, n.2, p.422-434. doi.org/10.28998/rpss.v2i2.3171

Soares, B.M; Campanholi, L.L. (2019). Fundamentos e práticas da fisioterapia [recurso eletrônico]. Ponta Grossa (PR): Atena Editora.

Sprung, C.L.; Lopes, S.S. (2016). Utilização de Técnicas da Medicina Tradicional Chinesa (MTC) para Analgesia da Cervicalgia em Adultos: Revisão Sistemática. Rev Bras Terap e Saúde, v.7, n.1, p.7-15. doi.org/10.7436/rbts-2015.07.01.02

Tavares, F., Chaves, T., Silva, E., Guerreiro, G., Gonçalves, J., \& Albuequerque. A. (2017). Efeitos imediatos da mobilização articular em relação à intervenção sham e controle na intensidade de dor e incapacidade em pacientes com dor lombar crônica: ensaio clínico aleatorizado controlado. Rev Dor. São Paulo, v.18.n.1, p.2-7. doi.org/10.5935/1806-0013.20170002

Uthaikhup, S., Assapun, J., Watcharasaksilp, K., \& Jull, G. (2017). Effectiveness of physiotherapy for seniors with recurrent headaches associated with neck pain and dysfunction: a randomized controlled trial. The spine journal : official journal of the North American Spine Society, 17(1), 46-55. doi.org/10.1016/j.spinee.2016.08.008 